\title{
Characterizing the next-generation matrix and basic reproduction number in ecological epidemiology
}

\author{
M. G. Roberts · J. A. P. Heesterbeek
}

Received: 14 July 2012 / Revised: 26 September 2012 / Published online: 20 October 2012 (C) Springer-Verlag Berlin Heidelberg 2012

\begin{abstract}
We address the interaction of ecological processes, such as consumerresource relationships and competition, and the epidemiology of infectious diseases spreading in ecosystems. Modelling such interactions seems essential to understand the dynamics of infectious agents in communities consisting of interacting host and non-host species. We show how the usual epidemiological next-generation matrix approach to characterize invasion into multi-host communities can be extended to calculate $\mathcal{R}_{0}$, and how this relates to the ecological community matrix. We then present two simple examples to illustrate this approach. The first of these is a model of the rinderpest, wildebeest, grass interaction, where our inferred dynamics qualitatively matches the observed phenomena that occurred after the eradication of rinderpest from the Serengeti ecosystem in the 1980s. The second example is a prey-predator system, where both species are hosts of the same pathogen. It is shown that regions for the parameter values exist where the two host species are only able to coexist when the pathogen is present to mediate the ecological interaction.
\end{abstract}

Keywords Epidemiological stability $\cdot$ Ecological stability $\cdot$ Infectious diseases

In honour and appreciation of our friend, colleague and teacher Odo Diekmann, on the occasion of his 65 th birthday.

M. G. Roberts $(\varangle)$

Institute of Information and Mathematical Sciences,

New Zealand Institute for Advanced Study, and Infectious Disease

Research Centre, Massey University, Private Bag 102 904, North Shore Mail Centre,

Auckland, New Zealand

e-mail: m.g.roberts@massey.ac.nz

J. A. P. Heesterbeek

Department of Farm Animal Health, Faculty of Veterinary Medicine, University of Utrecht, Yalelaan 7, 3584 CL Utrecht, The Netherlands 


\section{Mathematics Subject Classification (2000) 92D30 - 92D40}

\section{Introduction}

Most studies where mathematical models have been used to describe the dynamics of infectious disease have focused on interactions between one infectious agent and one host species. Studies involving more species have usually been restricted to those that are hosts of the same agent. Even if more species are involved, the interactions between individuals in the models have been focussed on those where transmission of the infectious agent could occur (which we will refer to as epidemiological interactions). Ecological interactions within and between species, such as consumer-resource relations (predator-prey and other feeding relations) and competition, were not traditionally explored in a systematic way when studying infection dynamics in a population. In the last five years, however, this situation has been changing in the theoretical literature. As more than $60 \%$ of newly emerging infections in humans originate from wildlife (Jones et al. 2008; Taylor et al. 2001), it is important to understand how infectious agents spread in wildlife communities, how they lead to changes in prevalence in some species rather than others, and how they can ultimately lead to increased, or even new, exposure to, and infections of, humans. Ecological field and experimental studies are starting to emerge that suggest infection dynamics in wildlife communities are influenced by ecological interactions in fundamental ways. Indeed, interactions with non-host species co-inhabiting the same ecosystems will influence infection dynamics in those species that are hosts.

We give a few motivating examples from the recent ecological literature. We do not claim to be exhaustive in coverage of the studies performed or the phenomena observed in the field - many more examples exist.

- Predators can be infected by pathogens and/or parasites that they share with prey via feeding, and the ecological interactions play a dominant role in determining the population effects of the infectious agent(s). For example: plague outbreaks (sudden epidemiological effect) and human hunting (gradual ecological effect) caused a decline in prairie dog numbers, possibly leading to the extinction in the wild of the black-footed ferret when this predator itself was experiencing canine distemper virus outbreaks; and plague in both predator and prey impede current reintroduction efforts (Matchett et al. 2010; Thorne and Williams 1988). Canine distemper virus is suggested to have been aggravated in lions in the Serengeti savannah ecosystem, in a cascade sparked by the drought-induced death of the lions, prey, buffalo, with tick-transmitted Babesia parasites in buffalo jumping species to lions (Munson et al. 2008).

- Manipulation of other species inhabiting the same ecosystem, whether host or nonhost, may change the prevalence of infectious agents in their main host species. For example: the prevalence of Lyme borreliosis in mice reacts to a reduction in abundance of non-competent species present in the same ecosystem that can act as a buffer (Keesing et al. 2010). The prevalence of Sin Nombre virus changes with ecosystem composition, including changes to non-host species (Dizney and Ruedas 2009; Súzan et al. 2009). There are indications of an inverse relationship 
between prevalence in host species and biodiversity in the ecosystem in which they live (Ostfeld et al. 2008), a dilution effect, but this is hotly debated (Randolph and Dobson 2012), once more emphasizing the need for a theoretical exploration of ecological and epidemiological interactions. Finally, Bartonella prevalence has been observed to increase in wood mice after the introduction of bank voles to the ecosystem (Telfer et al. 2005).

- The introduction or elimination of an infectious agent can lead to changes in ecosystem composition and balance. For example: rinderpest probably played an important role in regulating the Serengeti savannah ecosystem by limiting growth of the wildebeest population. After its eradication, an ecosystem change was observed in that tree cover in the Serengeti increased. This could be the result of an ecological (trophic) cascade: wildebeest population size increased, leading to increased consumption of grass, leading to reduced grass biomass and fewer fires fueled by dry grass. Fewer fires meant more opportunity for tree seedlings to mature (Holdo et al. 2009). Another example is the correlation of nematode parasite burden in grouse with predation risk (Redpath et al. 2006).

It seems essential therefore that one should explore ecological interactions in epidemiological models for infectious agents in multi-species systems. In the literature there has been substantial attention to simple predator-prey type models with a pathogen or parasite infecting either the prey, the predator, or both; with the infectious agents affecting notably survival and/or reproduction of their host. Depending on the modelling choices made, infectious agents have been shown to be able to both stabilize and destabilize predator-prey interactions, as well as having regulatory and other conservation consequences and effects on the spatial patterning of populations (Chattopadhyay and Arino 1999; Fenton and Rands 2006; Han et al. 2001; Han and Pugliese 2009; Haque and Venturino 2006; Hilker et al. 2007, 2009; Hilker and Malchow 2006; Hsieh and Hsiao 2008; Kacha et al. 2009; Malchow et al. 2005; Morozov 2011; Sieber et al. 2007; Siekmann et al. 2010; Venturino 1994, 1995, 2002). In an early contribution, Hadeler and Freedman analysed a predator-prey model with an infectious agent infecting both trophic levels, but without transmission within the levels. One could think of one species acting as intermediate host for a helminth parasite that is modelled as being indirectly transmitted. The model showed that the parasite could mediate coexistence of the prey and predator (Hadeler and Freedman 1989), as in our later example (Sect. 4). Venturino, who was one of the first to recognize the importance of this area of study, started a wave of attention by analysing various variants of Lotka-Volterra systems with infection (Venturino 1994). Beltrami and Carroll, at about the same time, used a simple model of a phytoplankton species (resource) and its grazer, with a virus infecting the resource and prohibiting infected phytoplankton cells from reproducing, to show that an infectious agent can be a strong regulator of population size (Beltrami and Carroll 1994). Several recent papers, notably by Hilker and co-workers (Hilker and Schmitz 2008; Oliveira and Hilker 2010) and by Venturino, Malkow and co-workers (Malchow et al. 2008), have provided overviews of the various studies since that pioneering work, and highlighted the differences between the models and their conclusions for epidemiological and ecological dynamics. It is not our aim here to review these results, or to address issues such as density vs 
frequency dependence in transmission, type of density dependence in growth, type of functional response of the resource, routes of transmission and effects of infection on hosts, course of infection in individual hosts (for example, infection life long or temporary, immunity life long or temporary, etc.), and the effects of heterogeneity in the host (for this see Malchow et al. 2008 and the references cited therein).

Most studies in the theoretical literature concentrate on an example model to derive analytical results for a particular case. There is a lack of more systematic approaches and general tools specific to eco-epidemiology. There have certainly been studies to address these issues conceptually (Hatcher et al. 2006), or to provide particular tools, for example concerning bifurcation patterns near $\mathcal{R}_{0}=1$ (Boldin 2006). In this paper, we contribute to a systematic approach by concentrating on the problem of invasion of an infectious agent. We demonstrate that at the infection-free steady state, the Jacobian matrix of a multi-host system described by ordinary differential equations is partitioned into ecological and epidemiological sub-matrices, and we provide an interpretation of these sub-matrices. Steady states may be unstable to ecological interactions, and/or unstable to invasion by a pathogen. We distinguish the ecological and epidemiological stability of steady states. We explore how the framework of the next generation matrix (NGM), introduced to characterize invasion of a susceptible population of species interacting epidemiologically by an infectious agent (Diekmann et al. 2010), can be extended to characterize invasion of a susceptible ecosystem of species, interacting both ecologically and epidemiologically. It is our aim to highlight how the next generation approach can be extended to this interaction between ecology and epidemiology, for which Ovide Arino may have been the first to coin the term eco-epidemiology (Chattopadhyay and Arino 1999; Malchow et al. 2008).

\section{A model for an infectious disease on a food web}

Consider $n$ species interacting in a food web. Let the population size of species $i$ be $N_{i}$, in an appropriate unit (population density, number of animals, biomass, etc.). Assume that the species have density-dependent per capita birth and death rates $v_{i}\left(N_{i}\right)$ and $\mu_{i}\left(N_{i}\right)$ respectively. Hence, species $i$ can sustain itself independently of all the other species if $v_{i}(0)>\mu_{i}(0)$. Let those species that are consumers of species $i$ have indices contained in the set $\mathcal{P}_{i}$, and those species that are consumed by species $i$ have indices contained in the set $\mathcal{Q}_{i}$. For simplicity of exposition we exclude cannibalism: $i \notin \mathcal{P}_{i} \cup \mathcal{Q}_{i}$; and assume that if species $i$ consumes species $j$, then species $j$ does not consume species $i: \mathcal{P}_{i} \cap \mathcal{Q}_{i}=\emptyset$. Both restrictions could be relaxed if necessary.

Suppose that species $i$ is consumed by species $j$, at a rate $\phi_{i j} N_{j}$, and species $j$ consequently increases its birth rate by $e_{j i} \phi_{j i} N_{i}$ (note $\phi_{j i}=\phi_{i j}$ ). Hence $e_{j i}$ is a measure of the efficiency of conversion of biomass of species $i$ into biomass of species $j$. We obtain the equations

$$
\frac{\mathrm{d} N_{i}}{\mathrm{~d} t}=\nu_{i}\left(N_{i}\right) N_{i}-\mu_{i}\left(N_{i}\right) N_{i}-N_{i} \sum_{j \in \mathcal{P}_{i}} \phi_{i j} N_{j}+N_{i} \sum_{k \in \mathcal{Q}_{i}} e_{i k} \phi_{i k} N_{k}
$$


for $i=1 \ldots n$. A steady state solution of the differential equation is $\left\{\bar{N}_{i}\right\}$, where either $\bar{N}_{i}=0$ or

$$
\mu_{i}\left(\bar{N}_{i}\right)-v_{i}\left(\bar{N}_{i}\right)=\sum_{j \in \mathcal{P}_{i}} \phi_{i j} \bar{N}_{j}-\sum_{k \in \mathcal{Q}_{i}} e_{i k} \phi_{i k} \bar{N}_{k}
$$

There are usually multiple steady states. The stability of each steady state may be deduced from the eigenvalues of the Jacobian matrix, $\mathbf{C}\left(\left\{\bar{N}_{i}\right\}\right)$, which has diagonal elements

$$
C_{i i}=\left[\left(v_{i}-\mu_{i}\right) \bar{N}_{i}\right]^{\prime}-\sum_{j \in \mathcal{P}_{i}} \phi_{i j} \bar{N}_{j}+\sum_{k \in \mathcal{Q}_{i}} e_{i k} \phi_{i k} \bar{N}_{k}
$$

where $\left[v_{i} \bar{N}_{i}\right]^{\prime}$ means the function $v_{i}\left(N_{i}\right) N_{i}$ differentiated with respect to $N_{i}$, and the result evaluated at $N_{i}=\bar{N}_{i}$. If $\bar{N}_{i}=0$ then $C_{i i}$ simplifies to $\nu_{i}(0)-\mu_{i}(0)-$ $\sum_{j \in \mathcal{P}_{i}} \phi_{i j} \bar{N}_{j}+\sum_{k \in \mathcal{Q}_{i}} e_{i k} \phi_{i k} \bar{N}_{k}$. The off-diagonal elements are $C_{i j}=-\phi_{i j} N_{i}$ for $j \in \mathcal{P}_{i}, C_{i k}=e_{i k} \phi_{i k} N_{i}$ for $k \in \mathcal{Q}_{i}$, and $C_{i \ell}=0$ for $\ell \notin \mathcal{P}_{i} \cup \mathcal{Q}_{i}$.

Let the food web be infected by a pathogen, with prevalence $I_{i} / N_{i}$ in species $i$ and frequency-dependent within-species transmission. Suppose that if species $i$ is consumed by species $j$, then the rate of consumption $\phi_{i j}$ is multiplied by $p_{i j}$ if the consumer is infected, and $q_{i j}$ if the resource is infected. Suppose also that infected members of species $i$ have a death rate increased by $\alpha_{i}$. The equations become

$$
\begin{aligned}
\frac{\mathrm{d} N_{i}}{\mathrm{~d} t}= & v_{i}\left(N_{i}\right) N_{i}-\mu_{i}\left(N_{i}\right) N_{i}-\alpha_{i} I_{i}-\sum_{j \in \mathcal{P}_{i}} \phi_{i j}\left(S_{i}+q_{i j} I_{i}\right)\left(S_{j}+p_{i j} I_{j}\right) \\
& +\sum_{k \in \mathcal{Q}_{i}} e_{i k} \phi_{i k}\left(S_{i}+p_{k i} I_{i}\right)\left(S_{k}+q_{k i} I_{k}\right)
\end{aligned}
$$

where $N_{i}=S_{i}+I_{i}$. In addition to within-species transmission, assume that all species contribute to a pool of environmental contamination when infected, and that they may also be infected from this pool. Assume also that a susceptible consumer may be infected by its resource during the act of consumption. The equations for the infected species population are

$$
\begin{aligned}
\frac{\mathrm{d} I_{i}}{\mathrm{~d} t}= & \beta_{i} \frac{S_{i} I_{i}}{N_{i}}-\mu_{i}\left(N_{i}\right) I_{i}-\alpha_{i} I_{i}-I_{i} \sum_{j \in \mathcal{P}_{i}} \phi_{i j} q_{i j}\left(S_{j}+p_{i j} I_{j}\right) \\
& +S_{i} \sum_{k \in \mathcal{Q}_{i}} c_{i k} \phi_{i k} I_{k}+\kappa_{i} S_{i} \sum_{\ell=1}^{n} r_{\ell} I_{\ell}
\end{aligned}
$$

The first summation is the loss of type $I_{i}$ due to consumption by other species, the second is the production of type $I_{i}$ due to hosts of type $S_{i}$ being infected during consumption, and the third is the infection of $S_{i}$ through environmental contamination. 
The Jacobian matrix is constructed from the diagonal components

$$
\frac{\partial}{\partial I_{i}}\left(\frac{\mathrm{d} I_{i}}{\mathrm{~d} t}\right)=\beta_{i} \frac{S_{i}-I_{i}}{N_{i}}-\mu_{i}\left(N_{i}\right)-\alpha_{i}-\sum_{j \in \mathcal{P}_{i}} \phi_{i j} q_{i j}\left(S_{j}+p_{i j} I_{j}\right)+r_{i} \kappa_{i}\left(S_{i}-I_{i}\right)
$$

and the off-diagonal components

$$
\begin{aligned}
\left.\frac{\partial}{\partial I_{j}}\left(\frac{\mathrm{d} I_{i}}{\mathrm{~d} t}\right)\right|_{j \in \mathcal{P}_{i}} & =I_{i} \phi_{i j} q_{i j}\left(1-p_{i j}\right)+\kappa_{i} S_{i} r_{j} \\
\left.\frac{\partial}{\partial I_{k}}\left(\frac{\mathrm{d} I_{i}}{\mathrm{~d} t}\right)\right|_{k \in \mathcal{Q}_{i}} & =S_{i} c_{i k} \phi_{i k}+\kappa_{i} S_{i} r_{k} \\
\left.\frac{\partial}{\partial I_{\ell}}\left(\frac{\mathrm{d} I_{i}}{\mathrm{~d} t}\right)\right|_{\ell \notin \mathcal{P}_{i} \cup \mathcal{Q}_{i}} & =r_{\ell} \kappa_{i} S_{i}
\end{aligned}
$$

The Jacobian matrix of the system at an infection-free steady state is therefore a $2 n \times 2 n$ matrix of the form

$$
\mathbf{J}=\left(\begin{array}{ll}
\mathbf{C} & \mathbf{D} \\
\mathbf{0} & \mathbf{H}
\end{array}\right)
$$

where $\mathbf{H}=\mathbf{T}+\boldsymbol{\Sigma}$ corresponds to the Jacobian matrix for the infection dynamics. The transmission matrix $\mathbf{T}$ has components

$$
T_{i j}= \begin{cases}\beta_{i}+r_{i} \kappa_{i} N_{i} & : i=j \\ r_{j} \kappa_{i} N_{i} & : j \in \mathcal{P}_{i} \\ c_{i j} \phi_{i j} N_{i}+r_{j} \kappa_{i} N_{i} & : j \in \mathcal{Q}_{i} \\ r_{i} \kappa_{i} N_{i} & : \text { otherwise }\end{cases}
$$

The transition matrix $\Sigma$ is diagonal, with entries

$$
\Sigma_{i i}=-\mu_{i}\left(N_{i}\right)-\alpha_{i}-\sum_{j \in \mathcal{P}_{i}} \phi_{i j} q_{i j} N_{j}
$$

We can say some more about the meaning of the different matrices constituting $\mathbf{J}$. First, the element $J_{i j}$ of $\mathbf{J}$ represent the sensitivity of the $i$ th variable to changes in the $j$ th variable, i.e. the elements of $\mathbf{J}$ describe the influence of the different variables on each other. Secondly, we have from an epidemiological point of view, chosen to phrase the exposition in terms of susceptible and infectious individuals only. Of course, there are several additional infected states (such as a latency state) and uninfected states (such as recovered and immune states) possible, in which individuals may behave differently from an epidemiological and ecological point of view. We ignore these in our exposition, in the interest of simplicity. In general one could set up the analysis with $n$ species and with $m$ infected states for individuals of these species, where $m$ may be smaller than $n$ (for example if not all species in the ecosystem are hosts of the infectious 
agent), or larger than $n$ (for example when there are additional relevant infectionrelated states for certain species beyond the infectious state). For our exposition we have chosen $n=m$.

Suppose we first regard the infection-free steady state as the basis of constructing $\mathbf{J}$, as described above. In that case, the eigenvalue problem decouples, i.e., the characteristic equation splits in a product of the characteristic equations for $\mathbf{C}$ and $\mathbf{H}$. The matrices $\mathbf{C}$ and $\mathbf{H}$ are necessarily square. Matrix $\mathbf{C}$ has dimension $n \times n$, and $\mathbf{H}$ has dimension $n \times n$ (but in general $m \times m$ ). Matrix $\mathbf{C}$ describes the influence of any species on other species in their common ecosystem in terms of their total population sizes $N_{i}, i=1, \ldots, n$, evaluated at the infection-free steady state. Therefore, C is the community matrix (McCann 2012), determining the ecological stability of the infection-free steady state, i.e., stability against perturbation in a subset of the $N_{i}$, measured by whether the spectral bound $s(\mathbf{C})<0$. Matrix $\mathbf{H}$ describes the influence of any infected state on other infected states. It is the Jacobian matrix of the infection sub-system, evaluated at the steady state for which it was constructed. Therefore, $\mathbf{H}$ is directly related to the so-called next-generation matrix with large domain in epidemic theory (introduced in Diekmann et al. 2010) $\mathbf{K}_{L}=-\mathbf{T} \boldsymbol{\Sigma}^{-1}$. The epidemiological stability of the infection-free steady state is governed by whether the spectral bound $s(\mathbf{H})<0$, or alternatively whether the spectral radius $\rho\left(\mathbf{K}_{L}\right)<1$ (see Diekmann et al. 2010). For other steady states, for example the endemic state with infection persisting in some or all of its host species, the eigenvalue problem does not decouple because the matrix in the bottom left corner (zero in the previous case) then contains non-zero elements. We will discuss an example in Sect. 4. For 'completeness' we note that for the cases studied in this paper, the matrix $\mathbf{K}_{L}$ coincides with the next-generation matrix $\mathbf{K}$ because the infected states coincide with the states-at-infection (Diekmann et al. 2010). In general, however, there will be more infected states than states-at-infection, and the matrix $\mathbf{H}$ will be related to $\mathbf{K}_{L}$. The spectral radii of $\mathbf{K}_{L}$ and $\mathbf{K}$ are equal, but the matrices are different in general (Diekmann et al. 2010).

The other two matrices constituting $\mathbf{J}$ are also square in our set up above, but will in general not be square. Their elements affect the ecological and epidemiological stability of the system as a whole whenever the matrix in the lower left corner is different from the 0-matrix. From an interpretation point of view, the elements of matrix $\mathbf{D}$ describe the additional influence of the infected states on the ecology of all species, expressed as their influence on the total population sizes $N_{i}$ of all species. One should, on the one hand, view individuals of a species $i$ in an infected state as similar to other individuals of species $i$, in their normal ecological interactions with others (of their own species and of any other species they are related to in the food web). These effects are incorporated in $\mathbf{C}$, where the infected individuals simply count as part of the total population size of species $i$. On the other hand, the fact that these particular individuals are in addition in an infected state can lead to additional effects on their own species' population size, and the population sizes of others, for example via changed feeding behavior or changes in reproductive ability. These influences in the total population size are measured by the elements of $\mathbf{D}$. The elements of the matrix in the lower left corner (zero in the exposition above), describe the additional ecological influence of the total population sizes of all species on the individuals in infected states. The influence of the infected part of the total population size, the 
epidemiological interaction, is incorporated in $\mathbf{H}$. The matrix in the lower left corner describes the additional effects of ecological interactions, for example changed density dependence acting on individuals in infected states.

\section{A resource - consumer/pathogen system}

This first example is motivated by the former rinderpest, wildebeest and grass interaction in the Serengeti. Rinderpest was a viral disease of ruminants with devastating consequences for cattle. In 2011 it became the second pathogen to be declared eradicated worldwide, after human smallpox in 1980. Intensive vaccination campaigns were aimed at domesticated ruminants that could seed outbreaks in wild animal populations, and led to eradication of the virus from wild animal populations, including wildebeest. It has been suggested (Holdo et al. 2009) that one side effect of the eradication of rinderpest in the Serengeti has been that fires have decreased in frequency, with increased survival of tree seedlings, due to the reduction in grass in the ecosystem. A possible reason for the latter is that the wildebeest population has grown explosively in the years following the eradication of rinderpest. This suggests that the virus had a strong regulatory effect on the wildebeest population, leading to a steady state with low levels of wildebeest and high levels of grass. Lifting the regulatory effect has allowed the wildebeest population to equilibrate at a new, higher level (see Fig. 4 in Holdo et al. 2009), with a concomitantly reduced level of grass.

Here, we designate wildebeest as the consumer and grass as its main resource. We have the following assumptions. The consumer population has density-dependent births; the consumer not only eats the resource but has an alternative source of lesser preference. The resource (grass) is assumed to show logistic growth. There is no infection in the resource. For simplicity we ignore a latency phase and assume that infected individuals are infectious for life, i.e. no exposed $E$ or recovered $R$ classes.

If there is no infection present, the equations are

$$
\begin{aligned}
\frac{\mathrm{d} N_{1}}{\mathrm{~d} t} & =\mu_{1} N_{1}\left(1-\frac{N_{1}}{K}\right)-\phi N_{1} N_{2} \\
\frac{\mathrm{d} N_{2}}{\mathrm{~d} t} & =v\left(N_{2}\right) N_{2}+e \phi N_{1} N_{2}-\mu_{2} N_{2}
\end{aligned}
$$

The function $v\left(N_{2}\right)$ describes the density dependence in consumer births, for the numerical examples we use $v\left(N_{2}\right)=\rho e^{-k N_{2}}$.

The steady state solutions are

1. The trivial steady state, $N_{1}=N_{2}=0$ is unstable.

2. The resource-only steady state, $N_{1}=K, N_{2}=0$, is unstable if $\nu(0)>\mu_{2}$ which we assume from now on.

3. The consumer-only steady state, $N_{1}=0, N_{2}=\hat{N}_{2}$, where $v\left(\hat{N}_{2}\right)=\mu_{2}$, is stable if $\phi \hat{N}_{2}>\mu_{1}$.

4. The coexistent steady state, $N_{1}=\bar{N}_{1}=K\left(1-\phi \bar{N}_{2} / \mu_{1}\right), N_{2}=\bar{N}_{2}$, solves $v\left(\bar{N}_{2}\right)=\mu_{2}-e \phi K\left(1-\phi \bar{N}_{2} / \mu_{1}\right)$, and is stable whenever it exists. By existence we mean that the steady state values of $N_{1}$ and $N_{2}$ are positive. When this is true 
$0<\bar{N}_{1}<K$ and $\hat{N}_{2}<\bar{N}_{2}<\mu_{1} / \phi$, hence the steady state $\left(0, \hat{N}_{2}\right)$ is unstable whenever the steady state $\left(\bar{N}_{1}, \bar{N}_{2}\right)$ exists.

When there is infection present, the system becomes:

$$
\begin{aligned}
\frac{\mathrm{d} N_{1}}{\mathrm{~d} t} & =\mu_{1} N_{1}\left(1-\frac{N_{1}}{K}\right)-\phi N_{1}\left(S_{2}+p I_{2}\right) \\
\frac{\mathrm{d} N_{2}}{\mathrm{~d} t} & =v\left(N_{2}\right) N_{2}+e \phi N_{1}\left(S_{2}+p I_{2}\right)-\mu_{2} N_{2}-\alpha I_{2} \\
\frac{\mathrm{d} I_{2}}{\mathrm{~d} t} & =\beta \frac{S_{2} I_{2}}{N_{2}}-\left(\mu_{2}+\alpha\right) I_{2}
\end{aligned}
$$

The Jacobian at any infection-free state $\left(N_{1}, N_{2}, 0\right)$ simplifies to

$$
\left(\begin{array}{ccc}
-\mu_{1} \frac{N_{1}}{K} & -\phi N_{1} & \phi(1-p) N_{1} \\
e \phi N_{2} & N_{2} v^{\prime}\left(N_{2}\right) & -e \phi(1-p) N_{1}-\alpha \\
0 & 0 & \beta-\mu_{2}-\alpha
\end{array}\right)
$$

The upper left $2 \times 2$ sub-matrix is the Jacobian matrix of the infection-free steady state, containing ecological interactions only. It determines the ecological stability of the steady state. The lower right $1 \times 1$ sub-matrix determines the stability of the infection-free steady state to invasion by the infectious agent. The steady state is epidemiologically unstable if $\mathcal{R}_{0}=\beta /\left(\mu_{2}+\alpha\right)>1$. The upper right $1 \times 2$ sub-matrix describes the effect of infected consumers on the consumer and resource population sizes. Therefore the structure of the Jacobian matrix decouples the eigenvalue problem for ecological stability from the problem for epidemiological stability. As shown above, the coexistent steady state $\left(\bar{N}_{1}, \bar{N}_{2}\right)$ of the ecological system is stable whenever it exists. Therefore a pathogen-free steady state solution $\left(\bar{N}_{1}, \bar{N}_{2}, 0\right)$ of the system, when it exists, is stable for $\mathcal{R}_{0}<1$ (no invasion possible), and unstable for $\mathcal{R}_{0}>1$.

For this example, we are interested to see if we can start from a stable steady state with infection, $\left(N_{1}^{*}, N_{2}^{*}, I_{2}^{*}\right)$, destabilize it by reducing $\mathcal{R}_{0}$ from greater than 1 to less than 1 , after which the system settles in a new stable steady state $\left(\bar{N}_{1}, \bar{N}_{2}, 0\right)$, with the population size of the consumer increased, and that of the resource decreased, compared to the steady state situation with infection present in the consumer population.

From the set of equations (Eq. 2), it follows that a steady state solution with consumer, resource and pathogen present satisfies

$$
\begin{aligned}
S_{2}^{*} & =\frac{N_{2}^{*}}{\mathcal{R}_{0}} \quad I_{2}^{*}=N_{2}^{*}-S_{2}^{*} \\
N_{1}^{*} & =K\left(1-\frac{\phi\left(S_{2}^{*}+p I_{2}^{*}\right)}{\mu_{1}}\right)=K\left(1-\frac{\phi r}{\mu_{1}} N_{2}^{*}\right) \\
v\left(N_{2}^{*}\right) & =\mu_{2}-e \phi N_{1}^{*}+\theta \frac{I_{2}^{*}}{N_{2}^{*}}
\end{aligned}
$$


where $\mathcal{R}_{0}>1, \theta=\alpha+e \phi(1-p) N_{1}^{*}$ and $r=p+(1-p) / \mathcal{R}_{0}<1$. When $N_{1}^{*}=0$, the set of equations (Eq. 3), has a solution $\left(0, \hat{N}_{2}^{*}, \hat{I}_{2}^{*}\right)$ whenever $v\left(\hat{N}_{2}^{*}\right)=$ $\left(\mu_{2}+\alpha\right)(1-\alpha / \beta)$ has a positive solution. That is, whenever

$$
v(0)>\left(\mu_{2}+\alpha\right)\left(1-\frac{\alpha}{\beta}\right)=\mu_{2}+\alpha\left(1-\frac{1}{\mathcal{R}_{0}}\right)>\lim _{N \rightarrow \infty} v(N)
$$

This represents a steady state with consumer and pathogen but no resource. Recall the assumption that the consumer has an alternative resource.

When $N_{1}^{*} \neq 0$ the steady state value of $N_{2}^{*}$ is the unique solution of

$$
v\left(N_{2}^{*}\right)=\left(\mu_{2}+\alpha\right)\left(1-\frac{\alpha}{\beta}\right)-e K \phi r\left(1-\frac{\phi r}{\mu_{1}} N_{2}^{*}\right)
$$

which exists when

$$
\begin{aligned}
v(0) & >\left(\mu_{2}+\alpha\right)\left(1-\frac{\alpha}{\beta}\right)-e K \phi r \\
& =\mu_{2}+\alpha\left(1-\frac{1}{\mathcal{R}_{0}}\right)-e K \phi\left(p+\frac{1-p}{\mathcal{R}_{0}}\right)>v\left(\frac{\mu_{1}}{\phi r}\right)
\end{aligned}
$$

The Jacobian matrix at either of these steady states $\left(N_{1}^{*}, N_{2}^{*}, I_{2}^{*}\right)$ simplifies to

$$
\mathbf{J}=\left(\begin{array}{ccc}
-\mu_{1} N_{1}^{*} / K & -\phi N_{1}^{*} & \phi(1-p) N_{1}^{*} \\
e \phi\left(S_{2}^{*}+p I_{2}^{*}\right) & \nu^{\prime}\left(N_{2}^{*}\right) N_{2}^{*}+\theta I_{2}^{*} / N_{2}^{*} & -\theta \\
0 & \beta I_{2}^{* 2} / N_{2}^{* 2} & -\beta I_{2}^{*} / N_{2}^{*}
\end{array}\right)
$$

For the steady state $\left(0, \hat{N}_{2}^{*}, \hat{I}_{2}^{*}\right)$, the first row of the matrix then consists entirely of zeros. Hence stability is determined by the $2 \times 2$ matrix

$$
\left(\begin{array}{cc}
v^{\prime}\left(\hat{N}_{2}^{*}\right) \hat{N}_{2}^{*}+\alpha \hat{I}_{2}^{*} / \hat{N}_{2}^{*} & -\alpha \\
\beta \hat{I}_{2}^{* 2} / \hat{N}_{2}^{* 2} & -\beta \hat{I}_{2}^{*} \hat{N}_{2}^{*}
\end{array}\right)
$$

which has negative trace and positive determinant. Hence, the steady state $\left(0, \hat{N}_{2}^{*}, \hat{I}_{2}^{*}\right)$ is stable whenever it exists.

Numerical results suggest that the endemic state $\left(N_{1}^{*}, N_{2}^{*}, I_{2}^{*}\right)$ is stable when it exists. A summary of these results is shown in Fig. 1. It can be seen that for $\mathcal{R}_{0}<1$ one of two pathogen-free steady states is attained, the consumer-only state $\left(0, \hat{N}_{2}, 0\right)$, or the coexistent state $\left(\bar{N}_{1}, \bar{N}_{2}, 0\right)$. These states lose ecological stability at the horizontal line, which is the critical value of $\phi$, and lose epidemiological stability at the vertical line $\mathcal{R}_{0}=1$. When the consumer-only steady state loses epidemiological stability, 


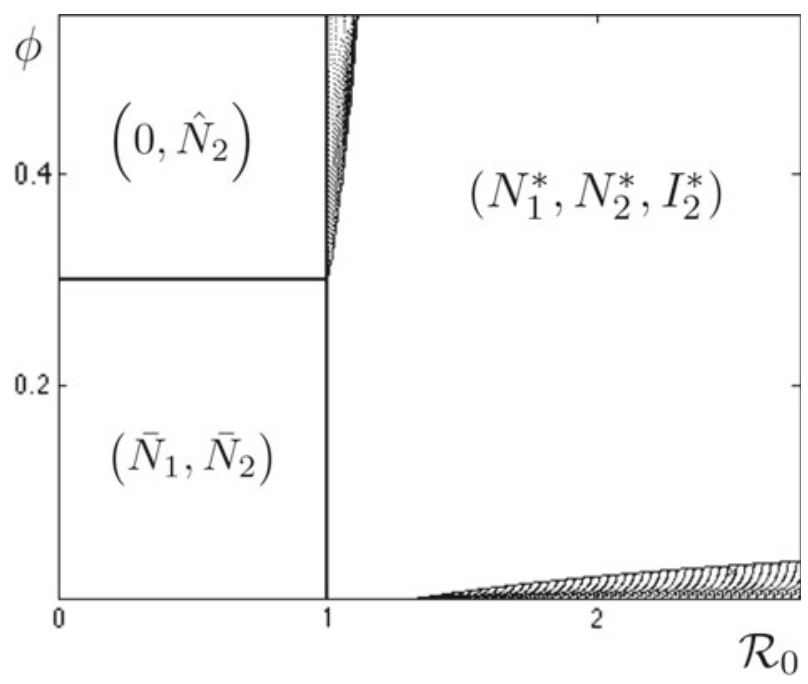

Fig. 1 Regions in the $\left(\mathcal{R}_{0}, \phi\right)$ plane showing where different steady states of the resource/consumer/ pathogen model exist and are stable. The regions labelled are: $\left(0, \hat{N}_{2}\right)$ and $\left(\bar{N}_{1}, \bar{N}_{2}\right)$, where the pathogen is absent; and the endemic state $\left(N_{1}^{*}, N_{2}^{*}, I_{2}^{*}\right)$. For more details see text. In the upper shaded region the steady state $\left(0, \hat{N}_{2}^{*}, \hat{I}_{2}^{*}\right)$ exists and is stable, in the lower shaded region the pathogen drives the consumer population to extinction. Parameter values are for illustration only: $\alpha=0.2, \mu_{1}=0.1, \mu_{2}=0.2$, $\rho=0.25, \beta=\mathcal{R}_{0}\left(\mu_{2}+\alpha\right)$ in units (time) ${ }^{-1} ; K=3$, (biomass); $k=0.6694$, (biomass) ${ }^{-1} ; \phi$ in units

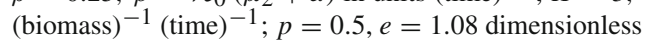

the steady state $\left(0, \hat{N}_{2}^{*}, \hat{I}_{2}^{*}\right)$ is attained. This state (the upper shaded wedge in Fig. 1) loses epidemiological stability at its right-hand boundary, to the resource, consumer, pathogen steady state $\left(N_{1}^{*}, N_{2}^{*}, I_{2}^{*}\right)$. It can also be seen in Fig. 1 that for high values of $\mathcal{R}_{0}$ and low values of the feeding rate $\phi$ it is possible that the pathogen drives the consumer to extinction.

Figure 2 shows putative bifurcation diagrams for this system. In Fig. 2a it is seen that at higher values of $\mathcal{R}_{0}$ the resource biomass approaches the carrying capacity in the absence of consumers, $K$. As the value of $\mathcal{R}_{0}$ decreases, so does the biomass $N_{1}^{*}$. In Fig. $2 \mathrm{~b}$ the increase in consumer density as $\mathcal{R}_{0}$ decreases is confirmed. This result illustrates how eradicating an infectious disease from a consumer population can increase population density, which at the same time reduces the biomass of a resource. For $\mathcal{R}_{0}<1$ the resource and consumer coexist in a sustainable equilibrium.

We conclude that this simple model is able to capture the processes essential to explain the observed dynamics in the Serengeti and reported in Holdo et al. (2009), moving from a situation with $\mathcal{R}_{0}>1$ to the current situation with $\mathcal{R}_{0}<1$. The data presented in Holdo et al. (2009) - Fig. 4, show a wildebeest population of around 200,000 animals in the ecosystem before 1960. An exponential increase in numbers started with the fast decline of rinderpest prevalence from about $80 \%$ before 1960 to close to $0 \%$ around 1965 , leading to equilibration around a new steady state of about 1,200,000 animals in 1980. This steady state has been more or less maintained since then. Of course, the actual ecological system is much more complicated, and 
Fig. 2 Steady state values of $\mathbf{a}$ the resource biomass and $\mathbf{b}$ the consumer population density as functions of the basic reproduction number rate $\mathcal{R}_{0}$. In a $\bar{N}_{1}$ and $N_{1}^{*}$ represent steady states of resource biomass in the presence of consumer only, and in the presence of consumer and pathogen respectively. In b $\bar{N}_{2}$ and $N_{2}^{*}$ are the corresponding values for the consumer population, $I_{2}^{*}$ is the steady state of the infected consumer population, and $\hat{N}_{2}$ is the steady state of the consumer population in the absence of resource and pathogen. For more details see text. Parameter values are for illustration only: $\alpha=0.2, \mu_{1}=$ $0.1, \mu_{2}=0.2, \rho=0.25$ in units (time) ${ }^{-1}$;

$K=3$, (biomass);

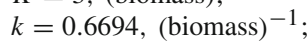

$\phi=0.0667$

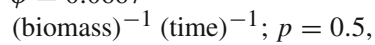
$e=1.08$ dimensionless
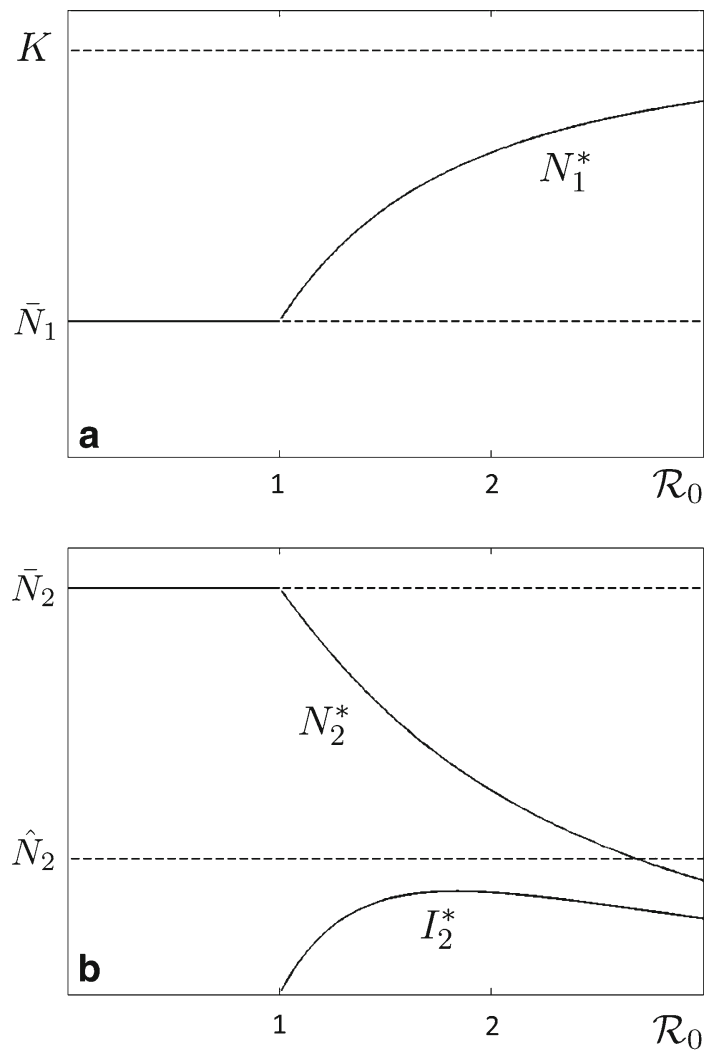

the change in $\mathcal{R}_{0}$ is simply a metaphor for a change in the wildebeest-rinderpest interaction that reduced the potential for wildebeest to become infected, but the basic dynamics are represented. Moreover, one can see from Fig. 1 that it is indeed the interplay between the strength of ecological interaction, here represented by feeding rate $\phi$, and the strength of epidemiological interaction, here represented by the basic reproduction number $\mathcal{R}_{0}$, that determines the dynamics and stability of the ecosystem consumer/resource/infectious agent.

\section{A prey-predator-pathogen system}

We now turn to a slightly more complicated example, where both the consumer and the resource are hosts to the infectious agent. As a biological motivation, think of a system with a predator (for example lions) and a resource (for example buffalo), sharing a pathogen (for example the bovine tuberculosis bacterium) that can be transmitted both within species or between species. Another example would be lions with zebra and anthrax, a pathogen that can also be transmitted via the environment (Getz 2011). Similar examples have previously been studied (Chattopadhyay and Arino 1999; Venturino 1994, 1995, 2002). 
Assume that the prey and predator have density-dependent birth rates. The predator not only eats the prey but has an alternative source of food. If there is no infection present, the equations are

$$
\begin{aligned}
& \frac{\mathrm{d} N_{1}}{\mathrm{~d} t}=v_{1}\left(N_{1}\right) N_{1}-\mu_{1} N_{1}-\phi N_{1} N_{2} \\
& \frac{\mathrm{d} N_{2}}{\mathrm{~d} t}=v_{2}\left(N_{2}\right) N_{2}+e \phi N_{1} N_{2}-\mu_{2} N_{2}
\end{aligned}
$$

In the explicit calculations we assume $v_{i}\left(N_{i}\right)=\rho_{i} e^{-k_{i} N_{i}}$. There are four steady state solutions: the trivial steady state, prey only, predator only and coexistence. The Jacobian matrix is

$$
\mathbf{C}=\left(\begin{array}{cc}
{\left[v_{1} N_{1}\right]^{\prime}-\mu_{1}-\phi N_{2}} & -\phi N_{1} \\
e \phi N_{2} & {\left[v_{2} N_{2}\right]^{\prime}+e \phi N_{1}-\mu_{2}}
\end{array}\right)
$$

Hence

1. The trivial steady state, $N_{1}=N_{2}=0$ is stable if $\nu_{1}(0)<\mu_{1}$ and $\nu_{2}(0)<\mu_{2}$.

2. The prey-only steady state, $N_{1}=\hat{N}_{1}, N_{2}=0$, where $v_{1}\left(\hat{N}_{1}\right)=\mu_{1}$, is stable if $\nu_{2}(0)+e \phi \hat{N}_{1}<\mu_{2}$.

3. The predator-only steady state, $N_{1}=0, N_{2}=\hat{N}_{2}$, where $\nu_{2}\left(\hat{N}_{2}\right)=\mu_{2}$, is stable if $v_{1}(0)<\mu_{1}+\phi \hat{N}_{2}$.

4. The coexistent steady state, $N_{1}=\bar{N}_{1}, N_{2}=\bar{N}_{2}$, solves $v_{1}\left(\bar{N}_{1}\right)=\mu_{1}+\phi \bar{N}_{2}$ and $\nu_{2}\left(\bar{N}_{2}\right)=\mu_{2}-e \phi \bar{N}_{1}$, and is stable whenever it exists.

In the above statements, stability refers to ecological stability. We now investigate the epidemiological stability of the system to the introduction of a pathogen that infects prey and predator. The infection routes are within species: prey to prey and predator to predator; prey to predator through feeding; and prey and predator to prey through the environment. We assume SIS infection dynamics in both hosts. Infected prey could be easier to catch $(q>1)$, or repulsive to the predator $(q<1)$. Infectious predators could be less likely to catch prey $(p<1)$. Substitute $N_{1}=S_{1}+I_{1}$ and $N_{2}=S_{2}+I_{2}$. The equations are:

$$
\begin{aligned}
\frac{\mathrm{d} N_{1}}{\mathrm{~d} t} & =v_{1}\left(N_{1}\right) N_{1}-\mu_{1} N_{1}-\alpha_{1} I_{1}-\phi\left(S_{1}+q I_{1}\right)\left(S_{2}+p I_{2}\right) \\
\frac{\mathrm{d} N_{2}}{\mathrm{~d} t} & =v_{2}\left(N_{2}\right) N_{2}+e \phi\left(S_{1}+q I_{1}\right)\left(S_{2}+p I_{2}\right)-\mu_{2} N_{2}-\alpha_{2} I_{2} \\
\frac{\mathrm{d} I_{1}}{\mathrm{~d} t} & =\beta_{1} \frac{S_{1} I_{1}}{N_{1}}-\left(\mu_{1}+\alpha_{1}\right) I_{1}-\phi q I_{1}\left(S_{2}+p I_{2}\right)+\kappa S_{1}\left(I_{1}+r I_{2}\right) \\
\frac{\mathrm{d} I_{2}}{\mathrm{~d} t} & =c q \phi I_{1} S_{2}+\beta_{2} \frac{S_{2} I_{2}}{N_{2}}-\left(\mu_{2}+\alpha_{2}\right) I_{2}
\end{aligned}
$$

Similar systems have previously been studied, see for example Hadeler and Freedman (1989) and Hsieh and Hsiao (2008). 
The Jacobian matrix at any disease-free state $S_{1}=N_{1}, S_{2}=N_{2}, I_{1}=I_{2}=0$, with $\left(N_{1}, N_{2}\right)=\left(\hat{N}_{1}, 0\right),\left(0, \hat{N}_{2}\right)$ or $\left(\bar{N}_{1}, \bar{N}_{2}\right)$, is of the form shown in Eq. 1 . The upper left $2 \times 2$ submatrix $\mathbf{C}$ is the Jacobian matrix of the infection-free system, as in Eq. 4. It determines the ecological stability of the steady state. The lower right $2 \times 2$ submatrix $\mathbf{H}=\mathbf{T}+\boldsymbol{\Sigma}$ determines the stability of the infection-free system to invasion by an infectious agent, that is the epidemiological stability. The matrix $\mathbf{T}$ contains the transmission terms, and $\boldsymbol{\Sigma}$ contains the transition terms.

$$
\mathbf{T}=\left(\begin{array}{cc}
\beta_{1}+\kappa N_{1} & r \kappa N_{1} \\
c \phi q N_{2} & \beta_{2}
\end{array}\right) \quad \boldsymbol{\Sigma}=-\left(\begin{array}{cc}
\mu_{1}+\alpha_{1} & 0 \\
0 & \mu_{2}+\alpha_{2}
\end{array}\right)
$$

The next-generation matrix is

$$
\mathbf{K}=-\mathbf{T} \Sigma^{-1}=\left(\begin{array}{cc}
\frac{\beta_{1}+\kappa N_{1}}{\mu_{1}+\alpha_{1}+\phi q N_{2}} & \frac{r \kappa N_{1}}{\mu_{2}+\alpha_{2}} \\
\frac{c \phi q N_{2}}{\mu_{1}+\alpha_{1}+\phi q N_{2}} & \frac{\beta_{2}}{\mu_{2}+\alpha_{2}}
\end{array}\right)
$$

and the steady state is epidemiologically unstable if $\mathcal{R}_{0}=\rho(\mathbf{K})>1$. If the two species exist in isolation of each other, then their basic reproduction numbers are the eigenvalues of $\mathbf{K}$ when $\phi=0$. Hence we write

$$
\left.\mathcal{R}_{0}^{1}=\frac{\beta_{1}+\kappa \hat{N}_{1}}{\mu_{1}+\alpha_{1}}\right) \quad \mathcal{R}_{0}^{2}=\frac{\beta_{2}}{\mu_{2}+\alpha_{2}}
$$

\subsection{Steady states with one species present}

We have seen that, in the absence of infection, the system has three non-trivial steady states, one each with a single species present, and a coexistent state. The ecological parameters of the system determine which state is realized and stable. We now consider the two possible steady states with a single species and infection present, and their stability.

\subsubsection{Prey and pathogen only}

The steady state solves $I_{1}^{*}=F_{1}\left(N_{1}^{*}\right) N_{1}^{*}=F_{2}\left(N_{1}^{*}\right) N_{1}^{*}$, where $F_{1}\left(N_{1}^{*}\right)=\left(v_{1}\left(N_{1}^{*}\right)-\right.$ $\left.\mu_{1}\right) / \alpha_{1}$ and $F_{2}\left(N_{1}^{*}\right)=1-\left(\mu_{1}+\alpha_{1}\right) /\left(\beta_{1}+\kappa N_{1}^{*}\right)$ with $N_{2}=I_{2}=0$. As $F_{1}(N)$ is a non-increasing function with $F_{1}\left(\hat{N}_{1}\right)=0$, then for this state to exist it is necessary that $\mathcal{R}_{0}^{1}>1$ and $F_{1}(0)>F_{2}(0)$. We then have a solution with $0<I_{1}^{*}<N_{1}^{*}<\hat{N}_{1}$. If $F_{1}(0)<F_{2}(0)$ then the pathogen is sufficiently virulent to drive the prey species to extinction. If the equations in Eq. 5 are reordered, swapping the second and third, the structure of the Jacobian at this steady state becomes 


$$
\mathbf{J}=\left(\begin{array}{cc}
\mathbf{H} & \mathbf{D} \\
\mathbf{0} & \mathbf{C}
\end{array}\right)
$$

The matrices that determine the stability of the steady state are then, after a little simplification

$$
\mathbf{H}=\left(\begin{array}{cc}
N_{1}^{*} v_{1}^{\prime}\left(N_{1}^{*}\right)+\alpha_{1} I_{1}^{*} / N_{1}^{*} & -\alpha_{1} \\
\kappa I_{1}^{*} & -\beta_{1} I_{1}^{*} / N_{1}^{*}
\end{array}\right)
$$

and

$$
\mathbf{C}=\left(\begin{array}{cc}
\nu_{2}(0)+e \phi\left(S_{1}^{*}+q I_{1}^{*}\right)-\mu_{2} & -e \phi(1-p)\left(S_{1}^{*}+q I_{1}^{*}\right)-\alpha_{2} \\
c q \phi I_{1}^{*} & \beta_{2}-\mu_{2}-\alpha_{2}
\end{array}\right)
$$

The matrix $\mathbf{H}$ controls the epidemiological stability of the system to the pathogen. It is always stable if $\beta_{1}>\alpha_{1}$, which follows from $\mathcal{R}_{0}^{1}>1$. The matrix $\mathbf{C}$ reflects the ecological stability of the system. That is, how it may be affected by introduction of the predator.

\subsubsection{Predator and pathogen only}

The steady state solves

$$
\frac{I_{2}^{*}}{N_{2}^{*}}=\frac{\nu_{2}\left(N_{2}^{*}\right)-\mu_{2}}{\alpha_{2}}=1-\frac{\mu_{2}+\alpha_{2}}{\beta_{2}}=1-\frac{1}{\mathcal{R}_{0}^{2}}
$$

with $N_{1}=I_{1}=0$. Arguing as above, existence of this state requires $\mathcal{R}_{0}^{2}>1$ and $\left(v_{2}(0)-\mu_{2}\right) / \alpha_{2}>1-1 / \mathcal{R}_{0}^{2}$, and leads to $0<I_{2}^{*}<N_{2}^{*}<\hat{N}_{2}$. If $\left(v_{2}(0)-\mu_{2}\right) / \alpha_{2}<1-1 / \mathcal{R}_{0}^{2}$ then the increased death rate from the pathogen is sufficient to drive the predator species to extinction. The Jacobian matrix at this steady state has the structure shown in Eq. 1. The eigenvalues of the Jacobian matrix are the eigenvalues of the matrices

$$
\mathbf{C}=\left(\begin{array}{cc}
\nu_{1}(0)-\mu_{1}-\phi\left(S_{2}^{*}+p I_{2}^{*}\right) & -\alpha_{1}-\phi q\left(S_{2}^{*}+p I_{2}^{*}\right) \\
r \kappa I_{2}^{*} & \beta_{1}-\mu_{1}-\alpha_{1}-\phi q\left(S_{2}^{*}+p I_{2}^{*}\right)
\end{array}\right)
$$

and

$$
\mathbf{H}=\left(\begin{array}{cc}
N_{2}^{*} \nu_{2}\left(N_{2}^{*}\right)^{\prime}+\alpha_{2} I_{2}^{*} / N_{2}^{*} & -\alpha_{2} \\
0 & -\beta_{2} I_{2}^{*} / N_{2}^{*}
\end{array}\right)
$$

which again determine ecological and epidemiological stability respectively. 


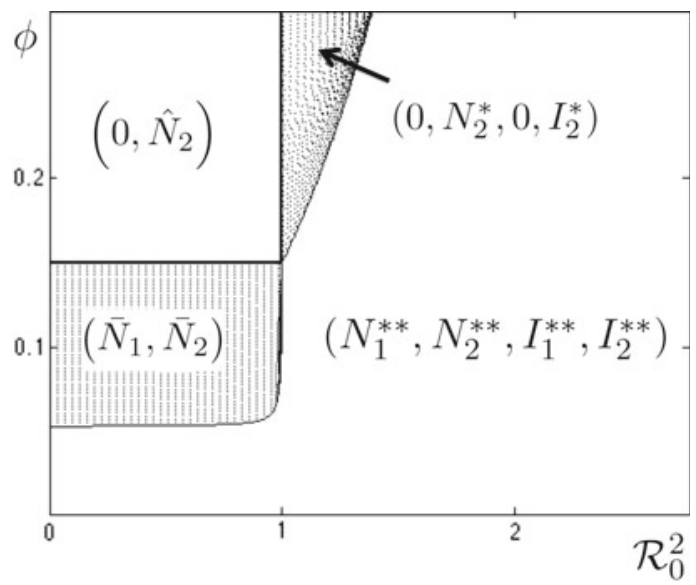

Fig. 3 Regions in the $\left(\mathcal{R}_{0}^{2}, \phi\right)$ plane showing where different steady states of the prey/predator/pathogen model exist and are stable. The regions labelled are: $\left(0, \hat{N}_{2}\right)$ and $\left(\bar{N}_{1}, \bar{N}_{2}\right)$, where the pathogen is absent; and the endemic states $\left(0, N_{2}^{*}, 0, I_{2}^{*}\right)$ where predator and pathogen are present, and $\left(N_{1}^{* *}, N_{2}^{* *}, I_{1}^{* *}, I_{2}^{* *}\right)$ where prey, predator and pathogen are present. For more details see text. Parameter values are for illustration only: $\alpha_{1}=0.02, \alpha_{2}=0.2, \beta_{1}=0.209, \beta_{2}=0.6, \mu_{1}=0.2, \mu_{2}=0.1, \rho_{1}=\rho_{2}=0.25$ in units [time] $^{-1} ; k_{1}=0.0744, k_{2}=2.7489$, [biomass] ${ }^{-1} ; \kappa=0.1$ [biomass] $^{-1}$ [time] $^{-1} ; p=0.5, q=r=$ $1, e=2.6185, c=0.1$ dimensionless

\subsection{Coexistent steady states_-prey, predator and pathogen present}

Numerical results confirm that for a region of parameter space the prey, predator and pathogen species coexist. The steady state values may be computed numerically, and confirmed by solving the differential equations over time and finding limiting values of the state variables. Figure 3 shows an example of the results plotted as regions in the $\left(\mathcal{R}_{0}^{2}, \phi\right)$ plane where different steady states exist and are stable. If $\mathcal{R}_{0}^{2}<1$, for values of the feeding rate $\phi$ above a critical value, only the predator population exists. The prey is driven to extinction. For lower values of $\phi$ both prey and predator species exist, but in the absence of pathogen. However, recall that $\mathcal{R}_{0}=\rho(\mathbf{K})$, the largest eigenvalue of the next-generation matrix. It can be seen from Eq. 6 that the leading diagonal element of $\mathbf{K}$ increases as $\phi$ decreases, and the trailing diagonal is $\mathcal{R}_{0}^{2}$. With the example parameters presented in Fig. 3, $\beta_{1} /\left(\mu_{1}+\alpha_{1}\right)=0.95$. The steady state value $\bar{N}_{1}$ is large for small $\phi$, hence for sufficiently low values of $\phi, \mathcal{R}_{0}>1$ even though $\mathcal{R}_{0}^{2}<1$ and the pathogen invades both species.

Another consequence of the results is shown in Fig. 4, where we illustrate a potential effect of the rate at which the predator consumes the prey on the coexistence of the system, and how the presence of the pathogen may mediate this. In Fig. 4a, horizontal broken lines show prey steady states, all in the absence of predators. The values are $\hat{N}_{1}>N_{1}^{*}>I_{1}^{*}$. Also shown in Fig. 4a are the corresponding prey steady states in the presence of predators, $\bar{N}_{1}$ (no pathogen present) and $\left(N_{1}^{* *}, I_{1}^{* *}\right)$, the steady state with pathogen. It can be seen in Fig. 4 a that for 
Fig. 4 Steady state values of $\mathbf{a}$ the prey population and $\mathbf{b}$ the predator population as functions of the consumption rate $\phi$. In a $\hat{N}_{1}, \bar{N}_{1}, N_{1}^{*}$ and $N_{1}^{* *}$ represent steady states of prey in the absence of predator and pathogen, in the presence of predator only, in the presence of pathogen only, in the presence of predator and pathogen respectively. $I_{1}^{*}$ and $I_{1}^{* *}$ are the corresponding steady state values of the infected prey population. In $\mathbf{b} \hat{N}_{2}, \bar{N}_{2}, N_{2}^{* *}$ and $I_{2}^{* *}$ are the corresponding values for the predator population. For more details see text. Parameter values are for illustration only: $\alpha_{1}=0.02$, $\alpha_{2}=0.2, \beta_{1}=0.209$, $\beta_{2}=0.6, \mu_{1}=0.2, \mu_{2}=0.1$, $\rho_{1}=\rho_{2}=0.25$ in units [time $^{-1} ; k_{1}=0.0744$, $k_{2}=2.7489$, [biomass] $^{-1}$; $\kappa=0.1$ [biomass] $^{-1}$ [time $^{-1}$; $p=0.5, q=r=1$, $e=2.6185, c=0.1$ dimensionless
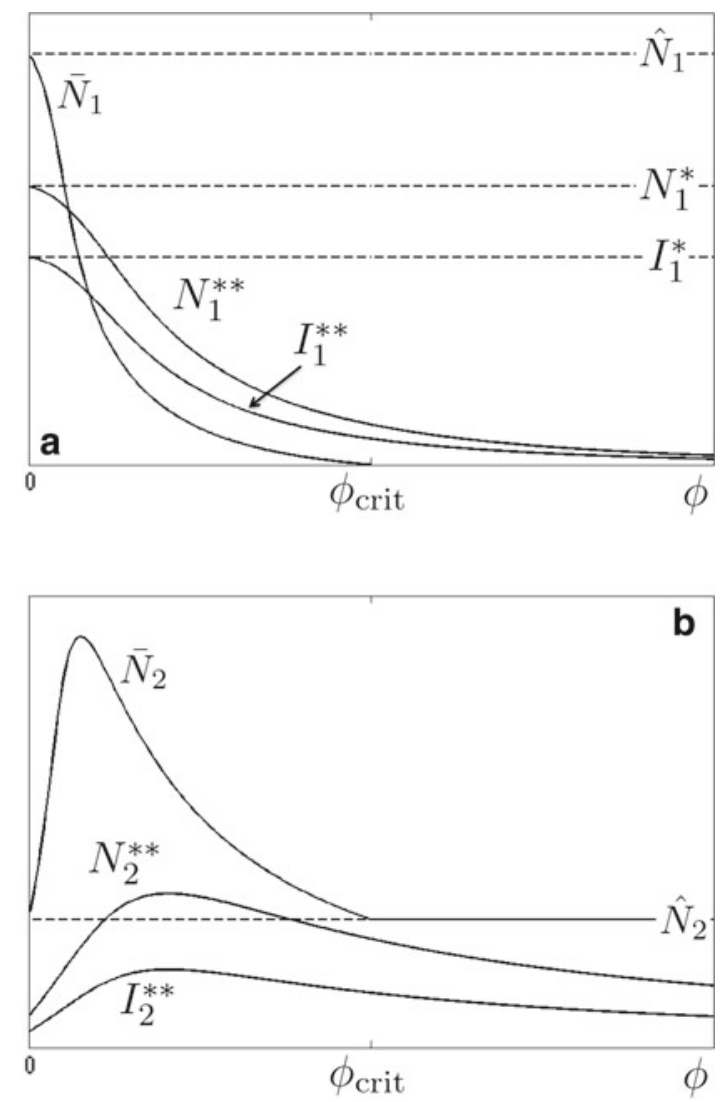

$\phi>\phi_{\text {crit }}$ the prey species cannot coexist with the predator, in the absence of the pathogen. The consumption rate is sufficiently high that the prey species is driven to extinction. If however, the pathogen is present, then all three species can coexist. This is because infection with the pathogen increases mortality in the predator population, reducing the population size and hence its impact on the prey population.

The effect of varying consumption rate on the predator population is shown in Fig. 4B. For $\phi<\phi_{\text {crit }}$ coexistence of predator and prey $\left(N_{2}=\bar{N}_{2}\right)$, or of predator, prey and pathogen $\left(N_{2}=N_{2}^{* *}, I_{2}=I_{2}^{* *}\right)$, is possible. However, for $\phi>\phi_{\text {crit }}$ coexistence of predator and prey is only possible in the presence of the pathogen.

\section{Discussion}

We have shown how one can characterize the invasion of in infectious agent into an ecosystem, and highlighted how this relates to stability analysis in both ecology and epidemiology. To do this we have extended the next-generation matrix approach to the basic reproduction number $\mathcal{R}_{0}$, as described in Diekmann et al. (2010). From a 
mathematical point of view, it will be interesting to derive new results concerning the interplay between ecological and epidemiological stability in the future.

Both of the example systems presented in Sects. 3 and 4, as well as the more general consumer-resource system in Sect. 2, reinforce a view that has been explored in many examples in the theoretical literature (see Sect. 1): ecological interactions not necessarily related to transmission events between a susceptible and an infectious individual, can strongly influence the dynamics of the infectious agent in the multi-species community to which the hosts belong. For our understanding of phenomena observed in real ecosystems and the interpretation of field data, either related to infectious disease prevalence in key species, or the composition, topology, balance and stability of ecosystems and food webs, it seems that such eco-epidemiological models could play an important role as additional tools of analysis. Clearly, much more work is needed, not only in relation to data, numerical exploration and biological inference, but also in generating tools for the mathematical analysis of these models. In addition, conceptual innovations are needed when thinking about incorporating various transmission routes and ecological relations between species into models; for example, contrasting various ways of exploring the transmission of infection via feeding, depending on the way energy or biomass flow is accounted for.

We believe that eco-epidemiological modeling, ultimately integrating ecosystem theory with epidemic theory, is an important challenge for theoretical biology and mathematics. The reason why such a theory can be fruitfully developed and explored now is that its development is best guided by field and experimental observations. Such results have been emerging only in recent years and are expected to become a major new branch of ecology in the future, with a widespread awareness that for an understanding of infectious diseases in natural populations, and perhaps even for an understanding of ecosystem dynamics itself, we need to consider multi-host/multiagent systems (Lafferty et al. 2006, 2008; Telfer et al. 2010).

Acknowledgments The authors thank two anonymous referees whose suggestions led to improvements in the manuscript. The first author received financial support from the Marsden Fund under contract MAU1106.

\section{References}

Beltrami E, Carroll TO (1994) Modelling the role of viral disease in recurrent phytoplankton blooms. J Math Biol 32:857-863

Boldin B (2006) Introducing a population into a steady community: the critical case, the centre manifold and the direction of bifurcation. SIAM J Appl Math 66:1424-1453

Chattopadhyay J, Arino O (1999) A predator prey model with disease in the prey. Nonlinear Anal 36:747766

Diekmann O, Heesterbeek JAP, Roberts MG (2010) The construction of next-generation matrices for compartmental epidemic systems. J R Soc Interface 7:873-885

Dizney LJ, Ruedas LA (2009) Increased host species diversity and decreased prevalence of Sin Nombre virus. Emerg Infect Dis 15:1012-1018

Fenton A, Rands SA (2006) The impact of parasite manipulation and predator foraging behavior on predatorprey communities. Ecology 87:2832-2841

Getz WM (2011) Biomass transformation webs provide a unified approach to consumer-reource modelling. Ecol Lett 14:113-124

Hadeler KP, Freedman HI (1989) Predator-prey populations with parasitic infection. J Math Biol 27: 609-631 
Han L, Ma Z, Hethcote HW (2001) Four predator prey models with infectious diseases. Math Comput Model 34:849-858

Han L, Pugliese A (2009) Epidemics in two competing species. Nonlinear Anal RWA 10:723-744

Haque M, Venturino E (2006) The role of transmissible diseases in Holling-Tanner predator-prey model. Theor Pop Biol 70:273-288

Hatcher MJ, Dick JTA, Dunn AM (2006) How parasites affect interactions between competitors and predators. Ecol Lett 9:1253-1271

Hilker F, Langlais M, Malchow H (2009) The Allee effect and infectious diseases: extinction, multistability, and the disappearance of oscillations. Am Nat 173:72-88

Hilker FM, Langlais M, Petrovskii SV, Malchow H (2007) A diffusive SI model with Allee effect and application to FIV. Math Biosci 206:61-80

Hilker FM, Malchow H (2006) Strange periodic attractors in a preypredator system with infected prey. Math Popul Stud 13:119-134

Hilker FM, Schmitz K (2008) Disease-induced stabilization of predator-prey oscillations. J Theor Biol 255:299-306

Holdo RM et al (2009) A disease-mediated trophic cascade in the Serengeti and its implications for ecosystem C. PLoS Biol 7(9):e1000210

Hsieh YH, Hsiao CK (2008) Predator-prey model with disease infection in both populations. Math Med Biol 25:247-266

Jones KE, Patel NG, Levy MA, Storeygard A, Balk D, Gittleman JL, Daszak P (2008) Global trends in emerging infectious diseases. Nature 451:990-993

Kacha A, Hbid MH (2009) Mathematical study of a bacteria model with level of infection structure. Nonlinear Anal Real World Appl 10:1662-1678

Keesing F et al (2010) Impacts of biodiversity on the emergence and transmission of infectious diseases. Nature 468:647-652

Lafferty KD et al (2006) Parasites dominate food web links. PNAS 103:11211-11216

Lafferty KD et al (2008) Parasites in food webs: the ultimate missing links. Ecol Lett 11:533-546

Malchow H, Petrovskii S, Venturino E (2008) Spatiotemporal patterns in Ecology and Epidemiology. Chapman and Hall/CRC, London

Malchow H, Hilker FM, Sarkar RR, Brauer K (2005) Spatiotemporal patterns in an excitable plankton system with lysogenic viral infection. Math Comput Modell 42:1035-1048

Matchett MR et al (2010) Enzootic Plague reduces black-footed ferret (Mustela nigripes) survival in Montana. Vector-Borne and Zoonotic Dis 10(1):27-35

McCann KS (2012) Food webs. Princeton University Press, Princeton

Morozov AYu (2011) Revealing the role of predator-dependent disease transmission in the epidemiology of a wildlife infection: a model study. Theor Ecol (published online 3 Nov 2011)

Munson L et al (2008) Climate extremes promote fatal co-infections during Canine distemper epidemics in African lions. PLoS One 3(6):e2545

Oliveira NF, Hilker (2010) Modelling disease introduction as biological control of invasive predators to preserve endangered prey. Bull Math Biol 72:444-468

Ostfeld RS, Keesing F, Eviner VT (eds) (2008) Infectious disease ecology: the effects of ecosystems on disease and of disease on ecosystems. Princeton University Press, Princeton

Randolph SE, Dobson ADM (2012) Pangloss revisited: a critique of the dilution effect and the biodiversitybuffers-disease paradigm. Parasitology (published online 16 Feb 2012)

Redpath SM et al (2006) Testing the role of parasites in driving the cyclic population dynamics of a gamebird. Ecol Lett 9:410-418

Sieber M, Malchow H, Schimansky-Geier L (2007) Constructive effects of environmental noise in an excitable preypredator plankton system with infected prey. Ecol Complex 4:223-233

Siekmann I, Malchow H, Venturino E (2010) On competition of predators and prey infection. Ecol Complex 7:446-457

Súzan G et al (2009) Experimental evidence for reduced rodent diversity causing increased hantavirus prevalence. PLoS One 4:e5461

Taylor LH, Latham SM, Woolhouse ME (2001) Risk factors for human disease emergence. Phil Trans R Soc B 356:983-989

Telfer S et al (2005) Disruption of a host-parasite system following the introduction of an exotic species. Parasitology 130:661-668 
Telfer S et al (2010) Species interactions in a parasite community drive infection risk in a wildlife population. Science 330:243-246

Thorne ET, Williams ES (1988) Disease and endangered species: the black-footed ferret as a recent example. Conserv Biol 2:66-74

Venturino E (1994) The influence of diseases on Lotka-Volterra systems. Rocky Mt J Math 24:381-402

Venturino E (1995) Epidemics in predator-prey models: disease among the prey. In: Arino O, Axelrod D, Kimmel M, Langlais M (eds) Mathematical population dynamics: analysis of heterogeneity, vol 1. Wuertz Publishing Ltd, Winnipeg, pp 381-393

Venturino E (2002) Epidemics in predator-prey models: disease in the predators. IMA J Math Appl Med Biol 19:185-205 